\title{
手性吲哚啉衍生物合成方法的研究进展
}

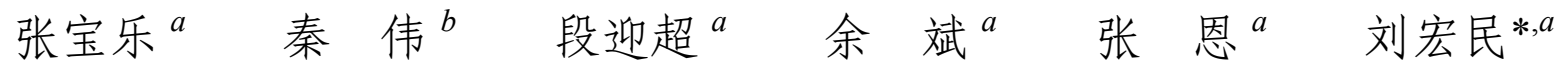 \\ ( ${ }^{a}$ 郑州大学药学院 郑州 450001) \\ $\left({ }^{b}\right.$ 齐齐哈尔医学院药学院 齐齐哈尔 161000)
}

\begin{abstract}
摘要 吲喍啉衍生物广泛存在于自然界中, 因具有多种生物学活性而引起广泛关注. 近年来, 手性吲哚啉衍生物成为 此领域的研究热点，也报道了多种相应的合成方法，主要包括动力学拆分、不对称合成、手性合成子策略等，不对称 Domino 级联反应作为近年来发展较快的领域, 也引起了广泛关注, 对以上合成方法加以综述.
\end{abstract}

关键词 吲哚啉; 不对称; 合成

\section{Recent Development on the Synthetic Methods of Chiral Indoline Derivatives}

\author{
Zhang, Baole $^{a} \quad{\text { Qin, } \mathrm{Wei}^{b}}$ Duan, Yingchao $^{a} \quad{\mathrm{Yu}, \mathrm{Bin}^{a}}^{a}$ Zhang, En $^{a} \quad$ Liu, Hongmin $^{* a}$ \\ ( ${ }^{a}$ School of Pharmaceutical Sciences, Zhengzhou University, Zhengzhou 450001) \\ ( ${ }^{b}$ School of Pharmacy, Qiqihar Medical University, Qiqihaer 161000)
}

\begin{abstract}
The indoline skeletons, widely present in alkaloids and other products with diverse biological activities, which also have been considered as privileged structures due to their widespread use as building blocks and chiral auxiliaries in asymmetric synthesis. The importance of these motifs has determined that numerous synthetic multi-step strategies have been developed. Consequently, there has been a continuing interest in the development of efficient methods to obtain enantioenriched substituted indolines. A lot of methods have been introduced, including dynamic kinetic resolution, asymmetric reactions, asymmetric domino cascade reactions and other novel reactions. These methods have been reviewed.
\end{abstract}

Keywords indoline; asymmetric; synthesis

吲哚及其衍生物是 Adolf von Baeyer 在 1866年首次 通过还原静红而获得, 1883 年, Fescher 和 Jourdan利用丙 酮酸和 1-甲基苯腙合成吲哚, 并于次年证实其结构 ${ }^{[1]}$. 吲哚啉结构作为一类具有生物活性的结构单元，普遍存 在于生物碱和其他天然产物中, 如 vinblastine (1), strychnine (2), (一)-physostigmine (3), ajmalinne (4) ${ }^{[2]}$ (图 1). 其中手性的 2-位取代和 2,3-位双取代的吲哚啉衍生 物因其广泛存在性和较好的生物活性而备受关注, 如 Benzasttain E (5)、血管紧张素酶抑制剂 Pentopril (6) ${ }^{[3]}$ (图 1). 此外, 吲哚啉作为手性辅助基也成功应用于有机 不对称合成中 ${ }^{[4]}$.

近十年有较多关于不对称合成和拆分吲哚啉衍生 物的文献报道, 也有文章对其进行综述 ${ }^{[3,5]}$. 主要包括氢
化还原、化学拆分、生物酶拆分、不对称合成、手性合 成子策略和不对称的 Domino 串联反应等. 本文主要对 合成手性吲哚啉衍生物的的方法加以概括.

\section{1 吲哚啉衍生物的动力学拆分}

动力学拆分是实现外消旋体拆分的一种有效方法, 加入的手性试剂和一对对映体反应生成非对映异构体, 因生成非对映异构体的活化能不同而具有不同的反应 速率, 利用不足量的手性试剂与外消旋体作用, 反应速 率快的对映体先完成反应，而留下反应速率慢的对映 体，从而达到拆分的目的.

\section{1 生物酶拆分法}

酶参与的动力学拆分因其环保性、操作简单和官能

\footnotetext{
*E-mail: liuhm@zzu.edu.com

Received November 29, 2011; revised January 21, 2012; published online March 31, 2012.

Project supported by the National Natural Science Foundation of China (No. 81172937 ), the China Postdoctoral Science Foundation (No. 20100480857), and the Special Financial Grant from the China Postdoctoral Science Foundation (No. 201104402).

国家自然科学基金(No. 81172937)、中国博士后科学基金(No. 20100480857)和中国博士后特别资助基金(No. 201104402)资助项目.
} 


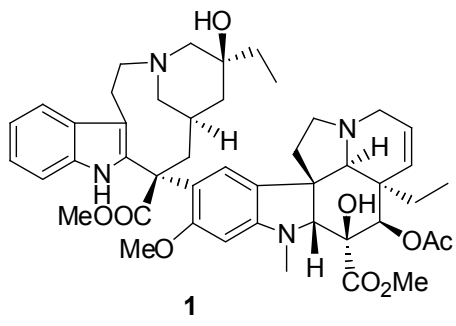

(+)-vinblastine

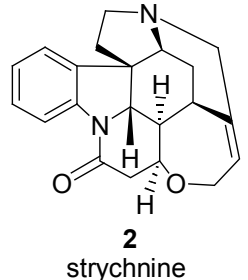

strychnine<smiles>CC(=O)Oc1ccc2c(c1)[C@@]1(C)CCN2CN1</smiles>

(-)-physostigmine

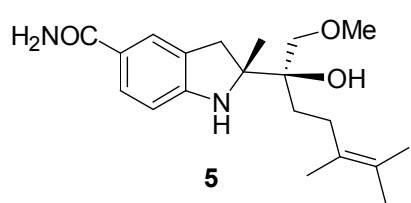

$(+)$-Benzastatin E

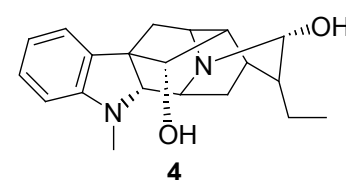

(+)-ajmaline

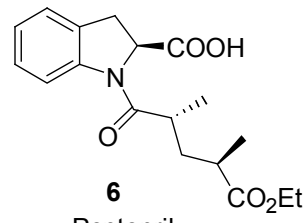

Pentopril
图 1 部分有生物活性含吲哚啉结构的化合物

Figure 1 Part of the bioactive indolines and their derivatives

才兼容性好而成为近年来研究较热的一个领域. 但吲哚 啉衍生物的外消旋体在用酶拆分时, 只有很少量的酶能 得到较好的 $e e$ 值, 而且在用枯草蛋白酶作生物催化剂 时虽 $e e$ 值很高, 但转化率较低 ${ }^{[3]} .1996$ 年, Wong 等 ${ }^{[6]}$ 首 次报道了通过脂肪酶和蛋白酶对 $\mathrm{N}$ 原子进行选择性的 酰化而实现 2-位取代吲哚啉 7 的动力学拆分的方法 (Scheme 1), 反应得到了较高的 $e e$ 值, 但是产率较低 (93\% ee, 6\% yield).

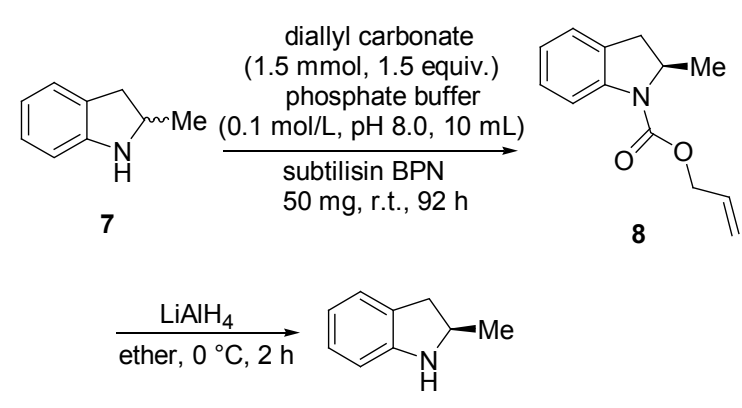

$(R)-9$

Scheme 1

2006 年, Gotor-Fernandez 等 ${ }^{[7]}$ 报道了南极洲脂肪酶 A (CAL-A)应用于 2-位取代吲哚啉衍生物外消旋体拆分 和南极洲脂肪酶 B (CAL-B) 对 3-位取代吲哚啉衍生物的 拆分. $30{ }^{\circ} \mathrm{C}$ 下, 以甲基叔丁基醚(TBME)为溶剂, 用酶 和羧酸烯丙酯 8 的混合物对 2-甲基吲哚啉外消旋体 7 进 行拆分(Eq. 1), 取得了较好的拆分效果 $(99 \% e e)$.<smiles>C=CCOC(=O)N1c2ccccc2CC1C</smiles>

尽管酶参与的动力学拆分有较多报道, 但由于其工 业化水平低和浓度增大时适应性差的缺点，限制了其实 际应用. 近年来手性辅助基催化的拆分取得了较大的进 展.

\section{2 化学拆分法}

Krasnov 等 ${ }^{[9]}$ 先后报道了以化合物 $\mathbf{1 0}$ 与 11 作为手 性试剂，与消旋体 2-甲基吲哚啉 7 发生酰化反应，经过 水解得到对映体 2-甲基吲哚啉 $(S)-9^{[8]}$ (76\% ee) 和 2-甲基 吲哚啉 $(R)-9(38 \%$ ee $)$ 的合成方法(Scheme 2).

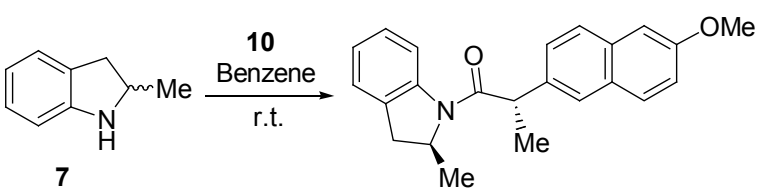

$(S, S)-1276 \%$ de

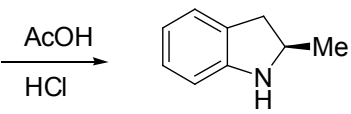

(R)-9 $76 \%$ ee<smiles>COc1ccc2cc([C@@H](C)C(=O)Cl)ccc2c1</smiles>

10<smiles>C[C@@H]1Cc2ccccc2N1C(=O)[C@@H]1CCCN1[As]</smiles>

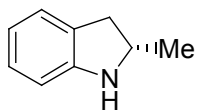

(S)-9 $38 \%$ ee

\section{Scheme 2}

2006 年, $\mathrm{Fu}$ 等 ${ }^{[10]}$ 报道了在手性二茂铁类化合物 $\mathbf{1 4}$ 催化下进行动力学拆分得到 $(R)-2$-取代吲哚啉的方法 (Eq. 2). 通过对影响拆分效果的因素分析后发现, 18-冠6 和 $\mathrm{LiBr}$ 存在下能取得较好的立体选择性, 2-位取代基 位阻较大有时拆分效果好 $(98 \% e e)$.

2009 年, Hou 等 ${ }^{[11]}$ 首次报道了金属 Pd 作催剂, 手性 试剂 15 催化 2-甲基取代吲哚啉 7 通过 $\mathrm{N}$ 原子烯丙基化 
进行拆分的方法(Eq. 3). 三乙胺或者碳酸钾的加入能提 高 $e e$ 值和转化率, 二氯甲烷(DCM) 作溶剂时拆分效果 好.
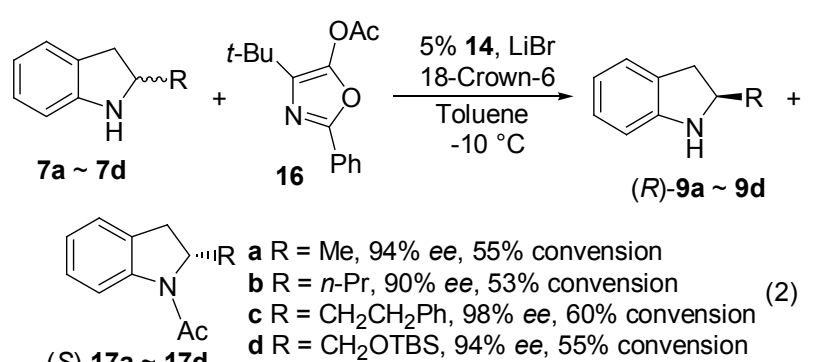

(S)-17a 17d
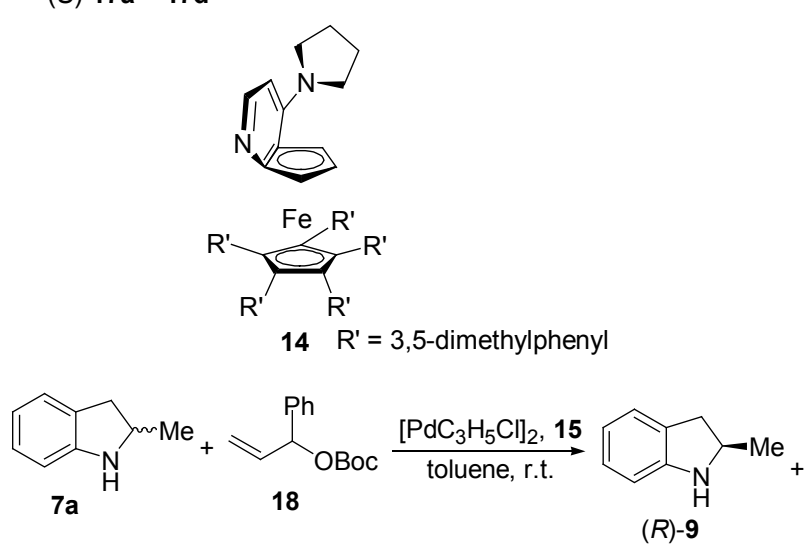<smiles>CC1Cc2ccccc2N1C/C=C/c1ccccc1</smiles>

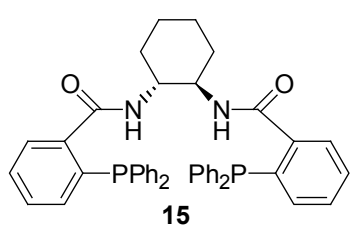

\section{2 通过不对称氢化反应合成吲哚啉衍生物}

在合成吲哚啉类化合物中, 通过对吲哚类化合物的 不对称氢化一直是研究较热的一个领域, 但因为吲哚结 构中双键和苯环的共轭结构而使还原条件非常苛刻, 而 且成本也因手性试剂的使用而升高, 故此类反应大多停 留在实验室方法学研究, 工业生产潜力不大.

2004 年, Kuwano 等 ${ }^{[12]}$ 首次报道了用铑作催化剂, 手性试剂 20 对五元的 $N$-乙酰基取代的吲哚衍生物 21 进行不对称氢化, 以大于 $95 \%$ 的 $e e$ 值得到光学纯的 2取代吲哚啉衍生物 $(R)-17$ 的方法 $(e e>95 \%$ ) (Scheme 3, Type 1). 不久, 他们 ${ }^{[13]}$ 又报道了 $N$-甲苯黄酰基取代和 3-位取代吲哚衍生物的选择性氢化方法( $>90 \% \mathrm{ee}$ ). $\left[\mathrm{Rh}(\mathrm{nbd})_{2}\right] \mathrm{SbF}_{6}$ 和手性双磷 PhTAP 络合物有效地催化了 反应的进行, $\mathrm{Cs}_{2} \mathrm{CO}_{3}$ 的加入有利于取得高的立体选择 性, 2-位取代的不同直接影响氢化产物的构型.

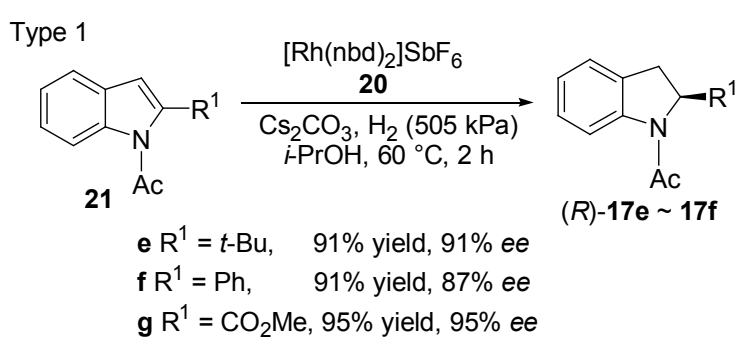

Type 2<smiles>[R]c1cc2ccccc2n1C(=O)OCc1ccccc1</smiles>

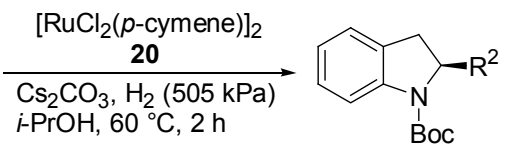

$22 a \sim 22 e$ a $\mathrm{R}^{2}=\mathrm{CO}_{2} \mathrm{Me}, 91 \%$ yield, $90 \%$ ee $23 a \sim 23 e$ b $\mathrm{R}^{2}=\mathrm{Me}, 99 \%$ yield, $95 \%$ ee c $\mathrm{R}^{2}=\mathrm{Bu}, 94 \%$ yield, $92 \%$ ee d $R^{2}=\mathrm{Ph}, 99 \%$ yield, $95 \%$ ee e $\mathrm{R}^{2}=c-\mathrm{C}_{6} \mathrm{H}_{11}, 92 \%$ yield, $87 \%$ ee<smiles></smiles>

$(S, S)-(R, R)-P h T R A P$

20

\section{Scheme 3}

两年后, Kuwano 课题组 ${ }^{[14]}$ 又报道了以手性钓为催 化剂([RuCl 2 (p-cymene) $]_{2}-(S, S)-(R, R)$-PhTRAP) 对 2-位取 代的 $N$-Boc 保护吲哚衍生物 22 的不对称氢化方法 (Scheme 3, Type 2).

2006 年, Chan 等 ${ }^{[15]}$ 报道了在 $\operatorname{Ir}(\mathrm{COD}) \mathrm{Cl}$ 作催化剂和 手性试剂 24 存在下, 对 2,3,3-三甲基取代吲哚(25)进行 不对称氢化得到化合物 $\mathbf{2 6}$ 的方法 $(72 \%$ ee) (Eq. 4).

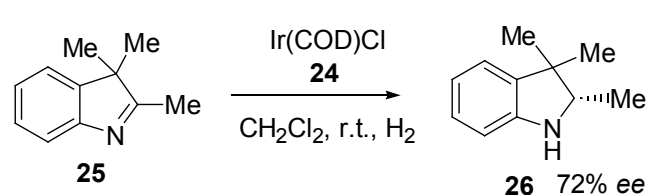<smiles>CC1C[C@H](C)Oc2cccc(-c3ccccc3)c2-c2c(cccc2-c2ccccc2)O1</smiles>

$(S, R, R)-\mathbf{2 4}$

2010 年, Baeza 和 Pfaltz 等 ${ }^{[16]}$ 报道了在金属铱盐化 合物 27 作催化剂下对 $N$-Boc 保护 2-甲基吲哚 22 催化氢 化得 2-甲基吲哚啉衍生物 23 (Eq. 5). 使用金属铱复合 体催化时不需要加入碱, 且催化剂对湿度和空气有更好 的稳定性, 故在不对称催化反应中有较大的应用前景. 


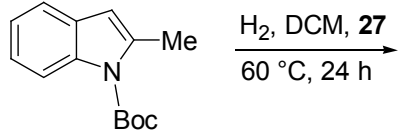

$22 b$

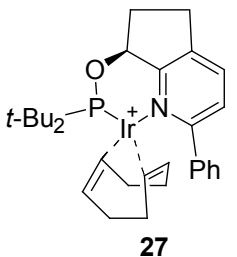

(5)

(R) Boc $89 \%$ conv. $99 \%$ ee

23b

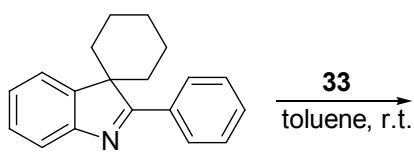

34

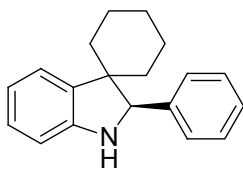

35 $98 \%$ yield, $97 \%$ ee

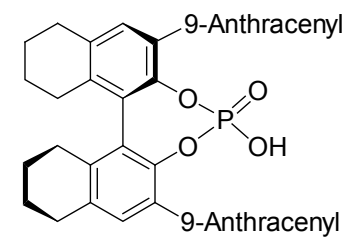

33

36 进行 3-位烷基化再进行不对称氢化得到 2,3-双取代的 吲哚啉衍生物 39 (ee>98\%) (Scheme 3).

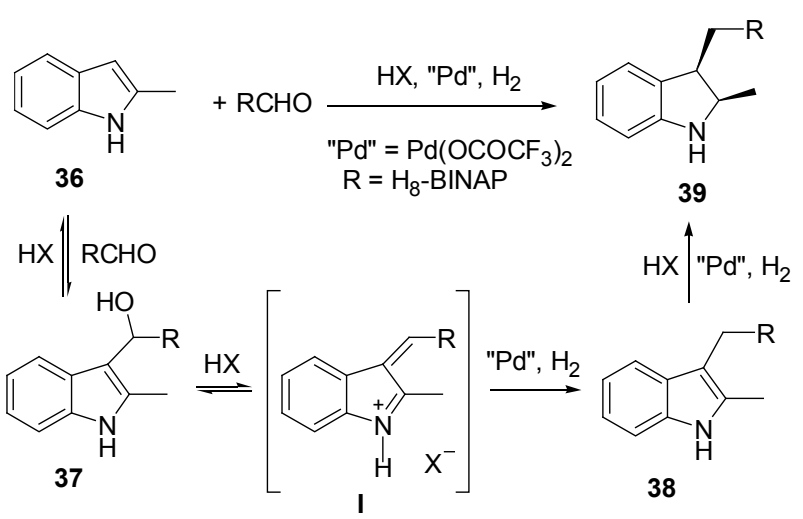

Scheme 3

\section{3 通过不对称合成法合成吲哚啉衍生物}

通过加入手性试剂的不对称合成法可以直接实现 手性合成且 $e e$ 值较高, 但是手性试剂价格昂贵一定程 度上限制了其应用.

\section{1 分子内缩合法}

2006 年, Yang 等 ${ }^{[21]}$ 报道了在 $(-)$-Sparteine (40)作手 性试剂和 $\operatorname{Pd}(\mathrm{II})$ 作催化剂下, 以化合物 41 为原料直接通 过氧化和分子内环合的级联反应来合成手性吲哚啉衍 生物 42 的方法(70\% yield, 86\% ee) (Eq. 9).

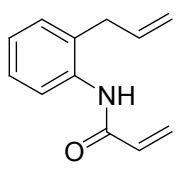

41

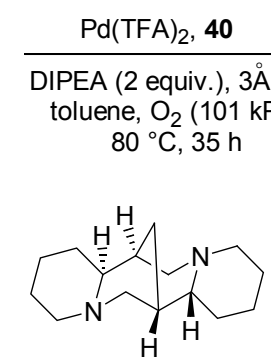

$(-)$-sparteine

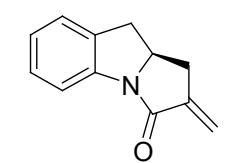

$4270 \%$ yield, $86 \%$ ee

40

2008 年, Chemler 等 ${ }^{[22]}$ 报道了以铜作催化剂, 反式- 
4,5-二苯基-双噁唑啉(43)为手性试剂, 通过在加热条件 下对苯胺类化合物 44 的双键氨基化不对称合成吲哚啉 衍生物 45 的方法(Eq. 10).
44

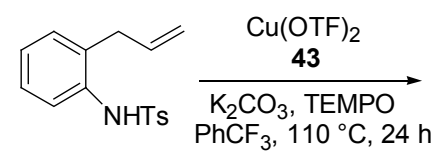

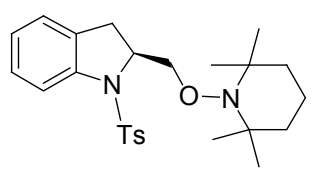

$4597 \%$ yield, $90 \%$ ee<smiles>CC(C)(C1=N[C@H](c2ccccc2)[C@H](c2ccccc2)O1)C1=N[C@H](c2ccccc2)[C@H](c2ccccc2)O1</smiles>

43
2010 年, Yamamoto 等 ${ }^{[23]}$ 首次报道了在 $\mathrm{Hg}(\mathrm{OTF})_{2}$ 催 化下通过对苯胺类化合物 46 分子内胺化环合来不对称 合成消旋的烯丙基取代吲哚啉衍生物 47 的方法, 副产 物只有水生成(99\% yield) (Scheme 4, Type 1). 当反应体 系中加入 $(R)$-BINAPHANE (48) 时, 化合物 46 能选择性 地合成 N-Ts 保护的 2-烯丙基取代吲哚啉 $(R)-47$ (99\% ee, $99 \%$ yield) (Scheme 4, Type 2).

Type 1<smiles></smiles>

46
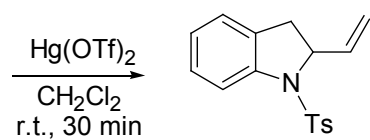

$4799 \%$ yield, racemate
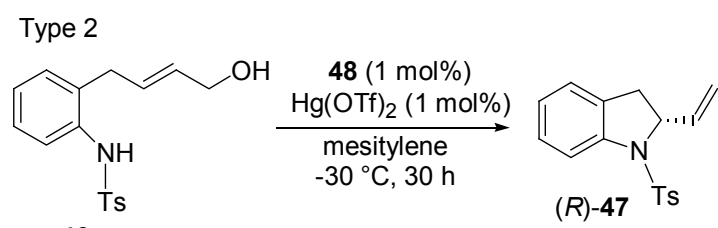

46 $99 \%$ yield, $99 \%$ ee

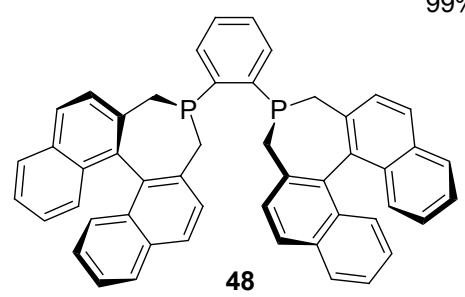

(R)-BINAPHANE

\section{Scheme 4}

2011 年, Nakanishi 等 ${ }^{[24]}$ 首次报道了通过手性氮杂 卡宾( $\mathrm{NHC}) 49$ 和 $\mathrm{Pd}$ 联合使用对化合物 $50 \mathrm{C}_{\mathrm{sp}^{3}}$ 直接烷基 化, 通过引入两个手性中心来合成光学活性的吲哚啉衍 生物 51 (Eq. 11). $\mathrm{Cs}_{2} \mathrm{CO}_{3}$ 的加入能提高立体选择性 (84\% yield, $e r=97.5: 2.5)$.

2011 年, Labadie 等 ${ }^{[25]}$ 首次报道了以硝基苯类化合 物 52 为原料通过 Diels-Alder 反应两步合成氮杂六氢咔

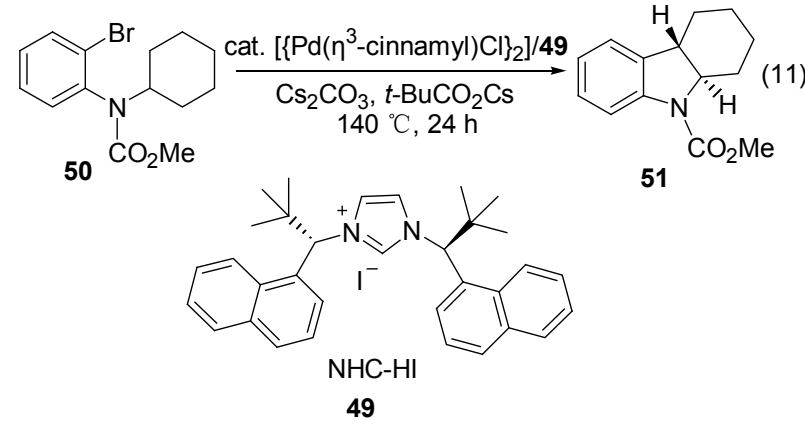

唑类吲哚啉衍生物 54 的方法(Scheme 5), 该反应官能团 兼容性好，起始原料易得，且通过核磁核间奥氏效应 (nuclear overhauser Effect, NOE)谱监测产物为顺势构型.

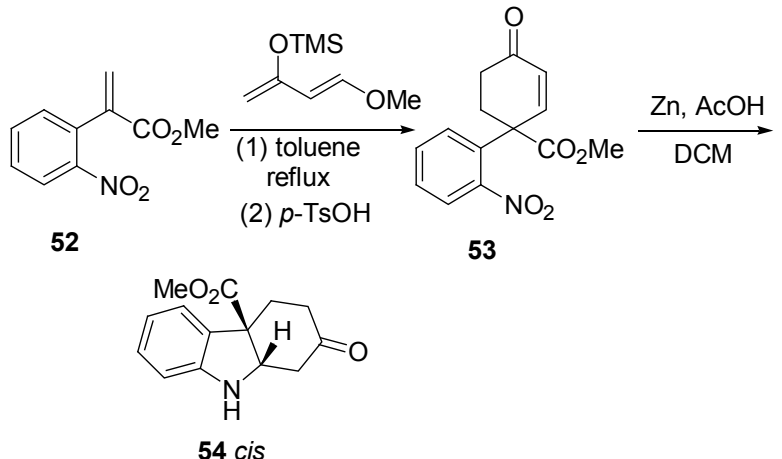

Scheme 5

2011, Hsung 等 ${ }^{[26]}$ 报道了一种新的分子内 DielsAlder 环化反应(IMDA), 是对 Oppolzer IMDA 的改进. 反应通过环己基苯胺类化合物 $\mathbf{5 5}$ 的烯胺异构化来合成 $\mathrm{N}$ 杂环类化合物 56 (30\% yield, $d r=2 ： 1)$ 和 $57(26 \%$ yield, $d r=2: 1$ ) (Scheme 6).
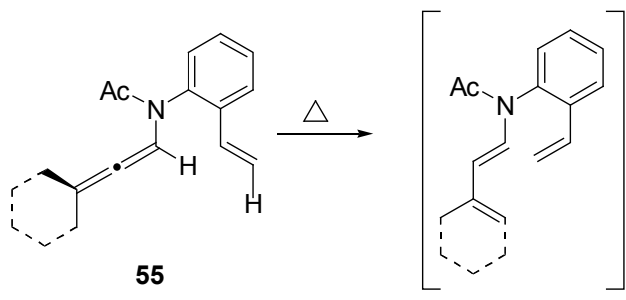

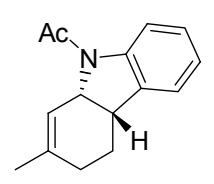

$5630 \%, d r=2: 1$

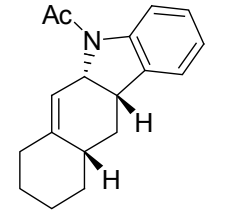

$5726 \%, d r=2: 1$
Scheme 6

\section{2 相转移催化法}

2003 年, Johnston 等 ${ }^{[27]}$ 报道了在相转移催化剂 $n-\mathrm{Bu}_{3} \mathrm{SnH}$ 和手性试剂 58 存在下，双溴代的芳香族化合 
物 59 和甘氨酸西弗碱 60 通过烷基化和胺化过程来合成 2-甲氧羰基取代的吲哚啉衍生物 61 的方法(96\% ee) (Scheme 7). 偶氮二异丁基腈(AIBN)的加入能提高反应 产率.

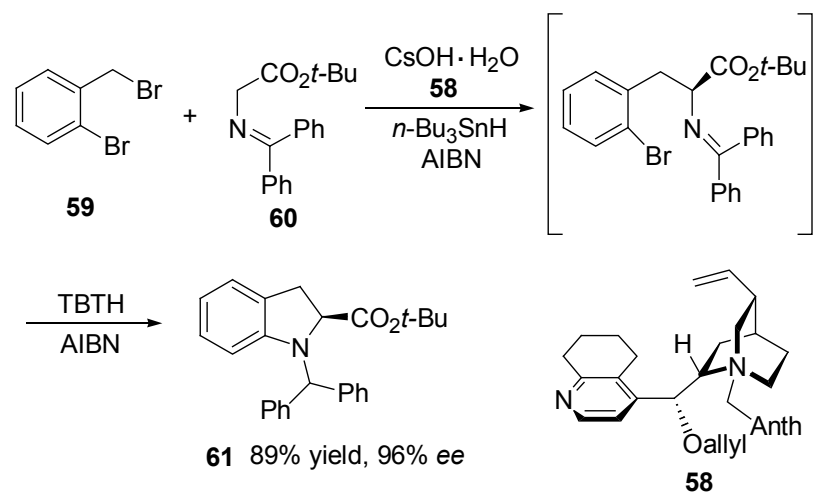

Scheme 7

2008 年, 该小组又报道了通过相转移催化的 Micheal 加成和芳胺化过程由化合物 62 合成 2,3-双取代 吲哚啉衍生物 63 的方法 ${ }^{[28]}$ (94\% yield, cis : trans $=86$ :

14) (Eq. 12).

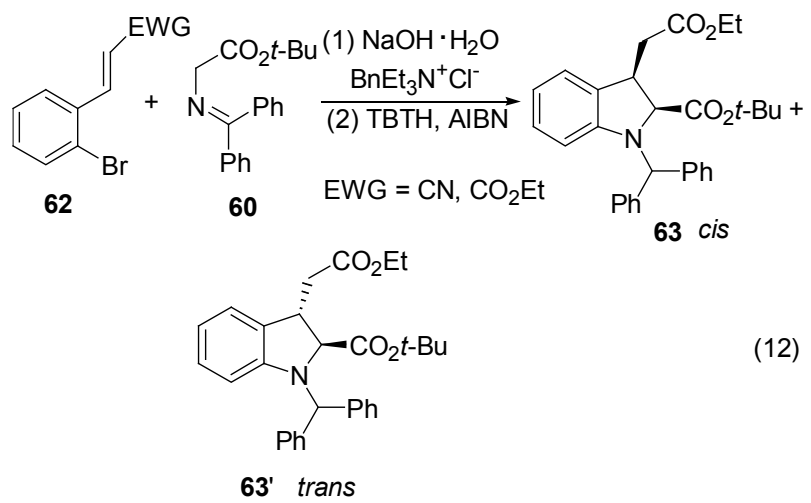

\section{3 电环化反应法}

2009 年, Maciver 等 ${ }^{[29]}$ 报道了在催化剂 64 存在下通 过 $6 \pi$ 体系的电环化反应由化合物 65 来合成吲哚啉衍生 物 66 的方法(Eq. 13). 环内外的取代基决了电环化反应 进行的程度和产物的立体选择性( $99 \%$ yield, $97 \% e e$ ).<smiles>[R]c1ccc(C(C(=O)OCC)C(=O)OCC)c(/N=C/c2ccccc2)c1</smiles>

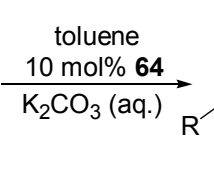<smiles>CCCOC(=O)C1(C(=O)OCC)c2ccccc2NC1c1ccccc1</smiles>

66

$\mathrm{R}=\mathrm{CF}_{3} 99 \%$ yield, $97 \%$ ee $\mathrm{R}=\mathrm{CH}_{3} 99 \%$ yield, $87 \%$ ee<smiles>[R2][C](c1ccccc1)C1CCC[N+]12CCCC2(C)C=C</smiles>

64

\section{4 烯丙基化法}

2005 年, Tamaru 等 ${ }^{[30]}$ 报道了在三乙基甲硼烷存在 下 Pd 催化 1-H 吲哚直接和烯丙醇反应通过在吲哚 3-位 引入烯丙基合成手性假吲哚(indolenine)的方法. 2006 年, Tros 等 ${ }^{[31]}$ 报道了在上述反应条件下以化合物 67 作为手 性试剂由 3-甲基吲哚类化合物 68 合成手性的 3-甲基-3烯丙基假吲哚 69 的方法(88\% yield, 83\% ee) (Eq. 14).

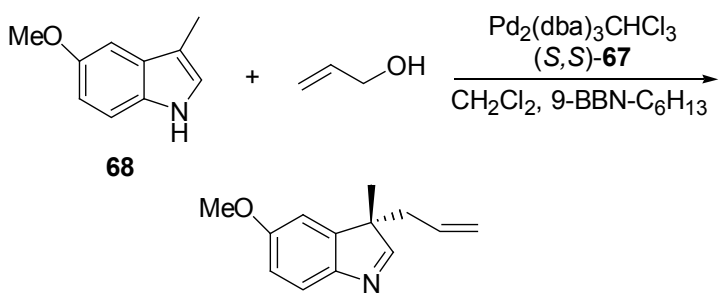

$6988 \%$ yield, $83 \%$ ee

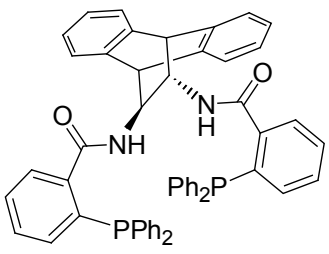

67

2010 年, Ttost 等 ${ }^{[32]}$ 报道了以 Mo 作催化剂, 手性试 剂 70 引导 3-苯基- $N$-甲基吲哚啉 2-酩(71) 不对称烯丙基 化(Asymmetric allylic alkylation, AAA), 生成 3,3-双取代 的吲哚啉 2-酩类化合物 72 和 73 的方法 $(d r=9: 1, e e=$ $92 \%)($ Eq. 15).
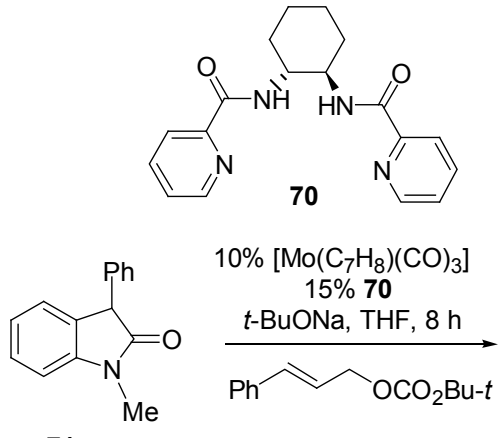

71<smiles>Cn1c(=O)n(C/C=C/c2ccccc2)c2ccccc21</smiles>

2011 年, Taylor 等 ${ }^{[33]}$ 报道了在 Pd 作催化剂和手性试 剂 74 存在下, 通过不对称的脱羰基来对 3-位烷基或 3芳基取代的吲哚啉 2-酮进行烯丙基化的方法. 即由丙二 酸 75 经中间体 76, 然后脱羰基得到 3-烷基-3-烯丙基取 代的吲哚啉 2-酮 77 (83\% yield, 74\% ee) (Scheme 8). 手 
性试剂的加入和取代基的大小都影响烯丙基化的立体 选择性.

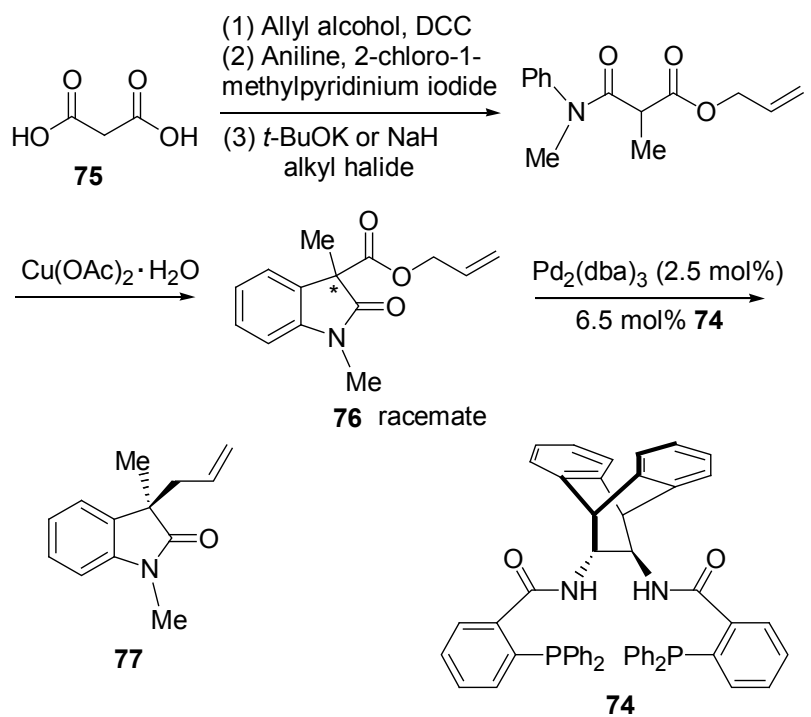

Scheme 8

\section{4 手性源对吲哚啉衍生物的选择性合成}

以手性化合物作为起始原料进行 2-位或 2,3-位双取 代吲哚啉衍生物的不对称合成因较好的选择性得到了 广泛的应用。

2002 年, Jackson 等 ${ }^{[34]}$ 报道了 Pd 催化有机锌试剂 79 和化合物临溴碘苯 $\mathbf{7 8}$ 经中间体 $\mathbf{8 0}$, 进而在 $\mathrm{Pd}$ 催化下环 化生成吲哚啉衍生物 23 , 取得了较好的转化率与对应 选择性(63\% 87\% yields, >99 \% ee) (Scheme 9).

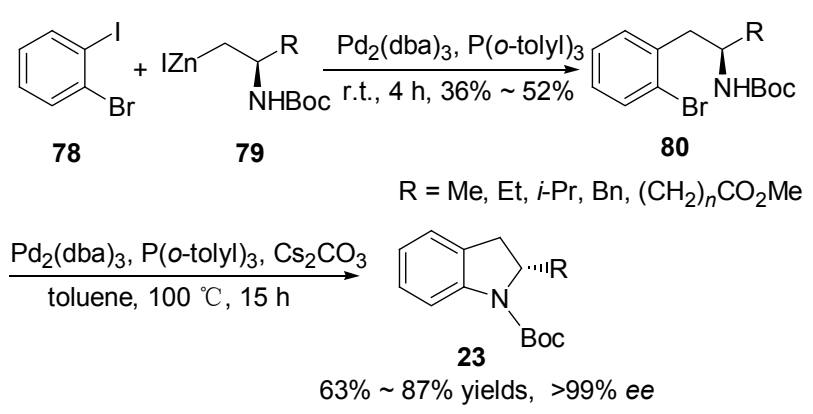

Scheme 9

2008 年, Buchwald 等 ${ }^{[35]}$ 报道了以铜为催化剂一步 不对称合成 2-取代吲哚啉衍生物的方法, 先以溴苯 81 和 $(S)$-1,2-环氧丁烷 $(82)$ 反应生成手性中间体 83 , 再和 Boc 氨 84 反应得到 $(R)$-2-乙基吲哚啉衍生物 23 (94\% yield, 99\% ee) (Scheme 10).

同一年, $\mathrm{Yu}$ 等 ${ }^{[36]}$ 报道了以手性氨基酸衍生物 85 为 起始原料, 首先在 $\mathrm{PhI}(\mathrm{OAc})_{2}$ 和 $\mathrm{I}_{2}$ 作用下, 生成二碘苯 中间体 86, 然后铜盐 $\mathrm{CuI}$ 催化分子内胺化来合成 2-甲氧 羰基取代的吲哚啉衍生物(S)-87 的方法(Scheme 11).

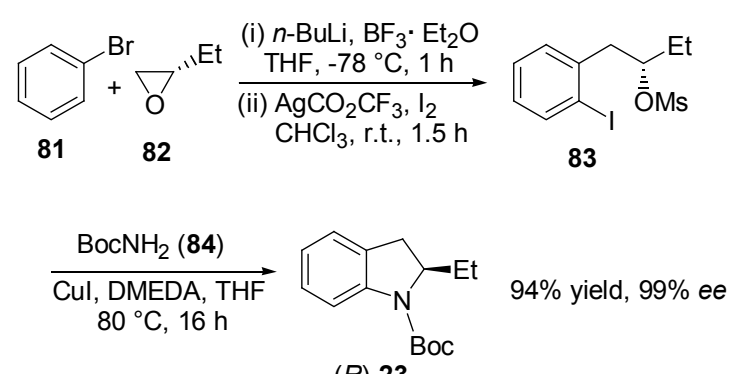

(R)-23

\section{Scheme 10}

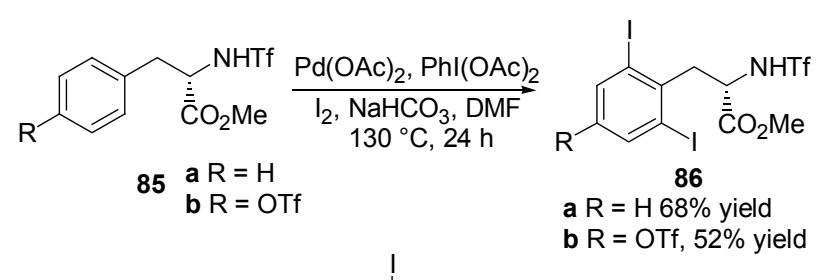

Cul, 1 equiv. $\mathrm{NaHCO}_{3}$ DMF, $130{ }^{\circ} \mathrm{C}, 24 \mathrm{~h}$

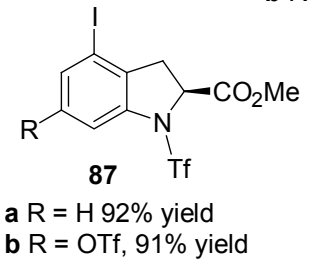

Scheme 11

2009 年, Curran 等 ${ }^{[37]}$ 报道了通过有手性轴的 2,4-二 甲基-6-碘代- $N$-烯丙基苯胺(88)环合得到 3-位羧甲酸酯 取代的哚啉衍生物 89 的方法(93\% yield, $e r=91 ： 9)$ (Eq. 16).

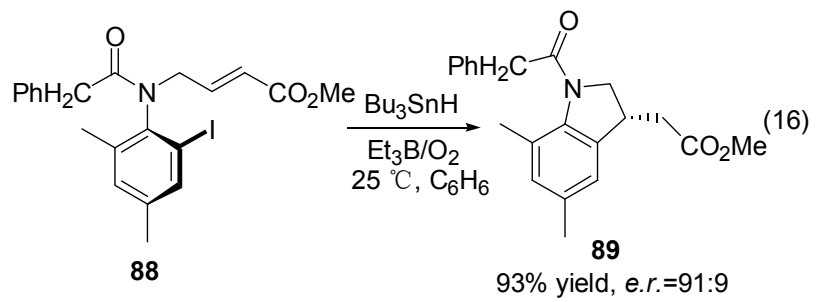

2011 年, Studer 等 ${ }^{[38]}$ 报道了一种通过手性源二碘代 苯胺类化合物 90 经自由基反应合成吲哚啉衍生物 91 的 方法(Scheme 12), 反应经历两种不同的途径即先还原 再环合或者先环合再还原，最后得到同一种产物( $86 \%$ yield, $d r=94: 6$ ).

2011 年, Andrade 等 ${ }^{[39]}$ 报道了全合成吲哚啉类天然 产物 Leuconicines A (9\% yield)和 B (10\% yield)的方法, 即以吲哚甲醛 92 为原料在手性辅助基 93 存在下选择性 合成吲哚衍生物 $94(d r=10: 1)$, 最后得到目标产物 (Scheme 13).

在获得手性吲哚啉类化合物方法中，化学拆分法发 展的时间较长、工艺相对成熟、但反应步骤多、污染较 严重、成本较高; 生物酶拆分法发展较迅速，但拆分酶 


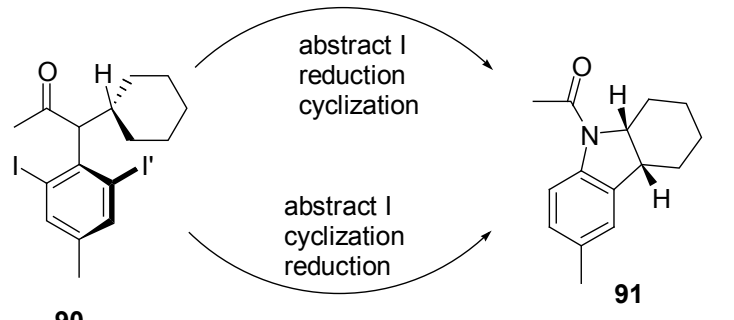

$86 \%$ yield, $d r=94: 6$

Scheme 12
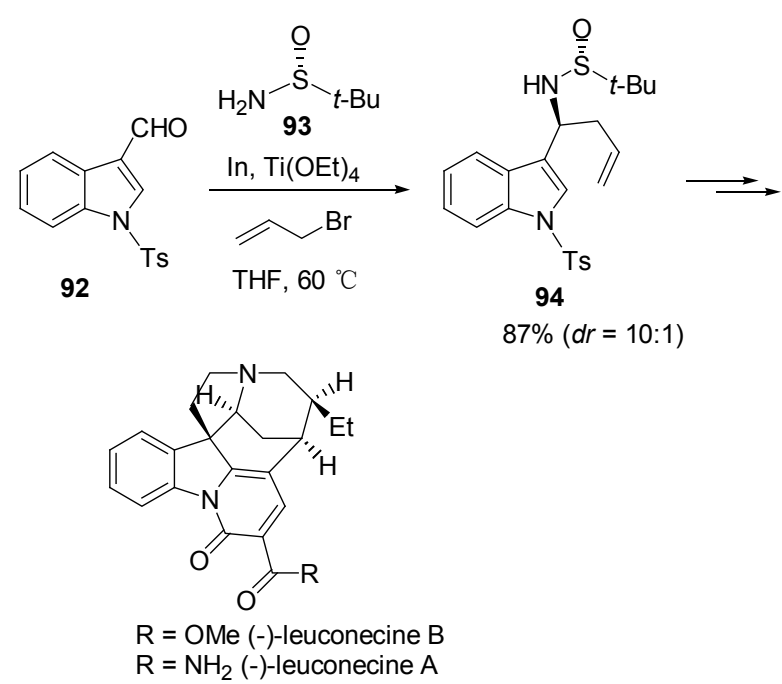

Scheme 13

种类缺乏、 $e e$ 值控制率低限制了其应用; 不对称合成法 收率和 $e e$ 值都较好, 但是手性试剂价格较昂贵; 手性源 合成法相比则显示出较好的应用前景.

\section{5 通过 Domino 级联反应来不对称合成吲哚啉 衍生物}

在有机合成中, Domino 串联反应通过一系列的成 键反应从简单化合物合成复杂化合物, 能减少有毒试剂 的使用. 最近也有 Domino 串联反应应用在吲哚啉衍生 物的不对称合成上, 因反应速率快、步骤和试剂用量少 而具有较好的应用前景 ${ }^{[40]}$.

2003 年, Kawasaki 等 ${ }^{[4]}$ 提出了以手性的 2-烯丙基氧 基吲哚啉-3-酮(95)为起始原料, 通过 domino 烯烃化和 不对称的 Claisen 重排来合成(一)-pseudophrynaminol (96) (97\% ee, 88\% yield)的方法(Scheme 14).

2008 年, Fustero 等 ${ }^{[42]}$ 报道了 2-甲苯磺酰基取代的 化合物 97 和亚胺化合物 98 经 $\mathrm{A}_{\mathrm{N}}$ 反应过程合成苯乙胺 类中间体 99 和 99', 分离中间体 99 后再通过 $\mathrm{S}_{\mathrm{N}} \mathrm{Ar}$ 级联 反应来合成光学纯的吲哚啉衍生物 $100(d r>98: 2$, yield $=50 \%$ ) (Scheme 15, Type 1). 当两步串联进行时环 合之前需将体系冷却到室温, 反应操作简化、条件温和
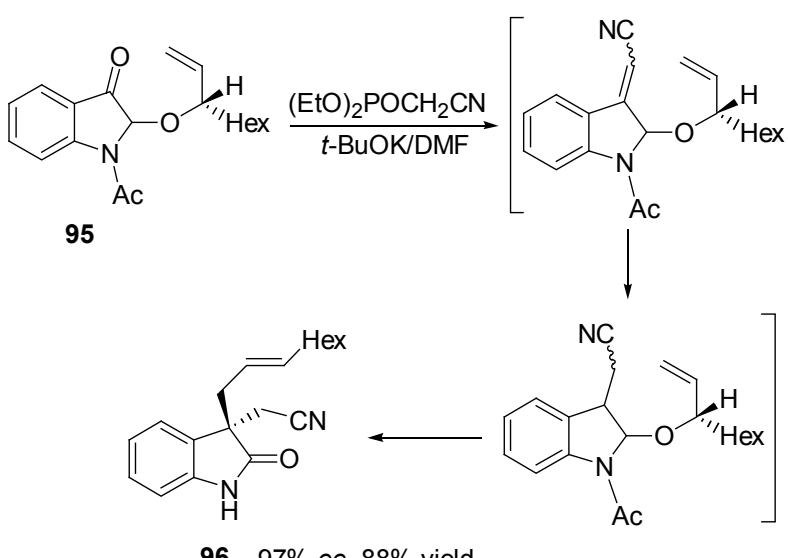

且非对应异构体比值 $(d r)$ 仍能保持 $(d r>98: 2$, 产率 55\%) (Scheme 15, Type 2).
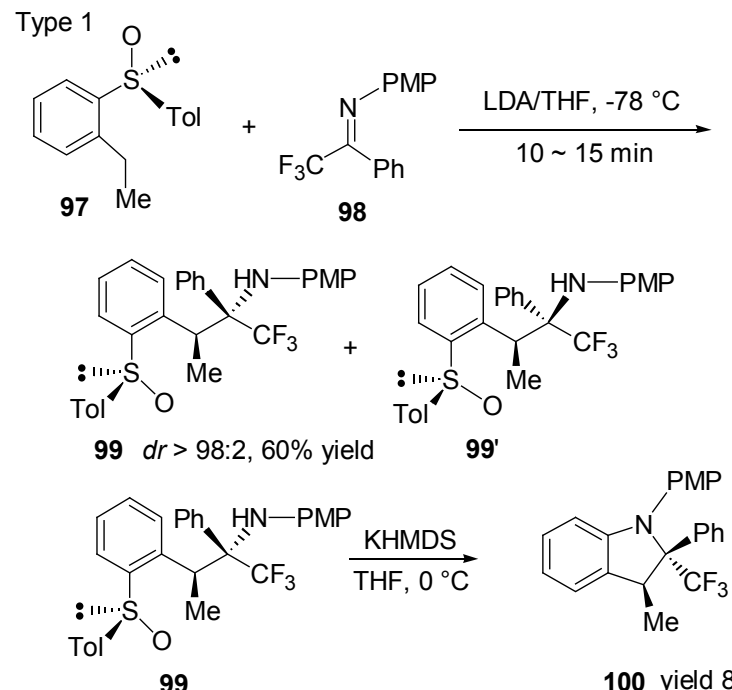

100 yield $83 \%$

Type 2

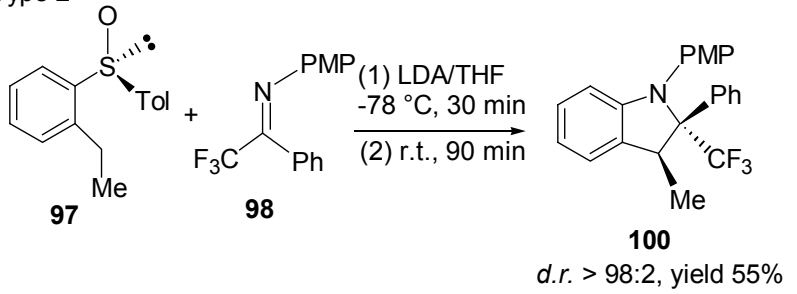

Scheme 15

2011 年，Yang 等 ${ }^{[43]}$ 报道了丙烯醛基苯胺类化合物 102 在 $\mathrm{Pd}(\mathrm{OAc})_{2}$ 嘧啶为催化剂, 等物质的量的氧气作为 绿色氧化剂和手性试剂 101 存在下发生级联反应合成吲 哚啉衍生物 103 的方法(64\% yield, 98\% ee) (Eq. 17).

\section{6 总结与展望}

由于光学纯取代吲哚啉衍生物的普遍存在和较好 的生物活性而引起化学家的广泛关注, 本文对其中一些 比较有代表性的方法和合成路线进行简单概括，希望能 


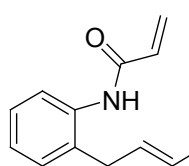

102

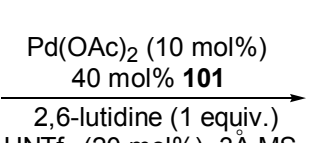

2,6-lutidine (1 equiv.) tolune, $\mathrm{O}_{2}(101 \mathrm{kPa}), 75^{\circ} \mathrm{C}$

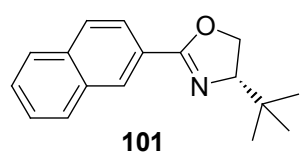

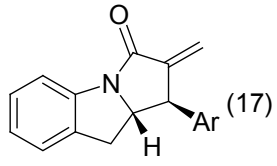

$103 d r>24: 1$ $98 \%$ ee
为不对称合成此类化合物提供一些依据, 并由此开拓出 更具创新性和实用性的合成方法. 由于不对称合成化学 中面临昂贵的手性试剂, 苛刻的反应条件, 反应收率低 等问题使其应用受到很大的限制. 最近发展起来的 Domino 串联反应在降低成本, 提高收率, 优化反应条 件方面都有很大的优势而在吲哚啉衍生物的不对称合 成中有较大的应用前景.

\section{References}

[1] Jiang, J.-Z.; Wang, Y. Chin. J. Org. Chem. 2006, 26, 1025 (in Chinese). (蒋金枝, 王艳, 有机化学, 2006, 26, 1025.)

[2] Liu, D.; Zhao, G.; Xiang, L. Eur. J. Org. Chem. 2010, 2010, 3975.

[3] Anas, S.; Kagan, H. B. Tetrahedron: Asymmetry 2009, 20, 2193.

[4] Jin, D.; Zhang, F.; Zhang, D.-C. Chin. J. Org. Chem. 2010, 30, 1005 (in Chinese). (金丹, 张峰, 张德纯, 有机化学, 2010, 30, 1005.)

[5] Liu, J.-Q.; Qian, C.; Chen, X.-Z. Chin. J. Org. Chem. 2011, 31, 634 (in Chinese). (刘金强, 钱超, 陈新志, 有机化学, 2011, 31, 634.)

[6] Orsat, B.; Alper, P. B.; Moree, W.; Mak, C. P.; Wong, C. H. J. Am. Chem. Soc. 1996, 118, 712.

[7] Gotor-Fernandez, V.; Fernandez-Torres, P.; Gotor, V. Tetrahedron: Asymmetry 2006, 17, 2558.

[8] Krasnov, V. P.; Levit, G. L.; Andreeva, I. N.; Grishakov, A. N.; Charushin, V. N.; Chupakhin, O. N. Mendeleev Commun. 2002, 12, 27.

[9] Krasnov, V. P.; Levit, G. L.; Bukrina, I. M.; Andreeva, I. N.; Sadretdinova, L. S.; Korolyova, M. A.; Kodess, M. I.; Charushin, V. N.; Chupakhin, O. N. Tetrahedron: Asymmetry 2003, 14, 1985.

[10] Arp, F. O.; Fu, G. C. J. Am. Chem. Soc. 2006, $128,14264$.

[11] Hou, X. L.; Zheng, B. H. Org. Lett. 2009, 11, 1789.

[12] Kuwano, R.; Kashiwabara, M.; Sato, K.; Ito, T.; Kaneda, K.; Ito, Y. Tetrahedron: Asymmetry 2006, 17, 521.

[13] Kuwano, R.; Kaneda, K.; Ito, T.; Sato, K.; Kurokawa, T.; Ito, Y. Org. Lett. 2004, 6, 2213.

[14] Kuwano, R.; Kashiwabara, M. Org. Lett. 2006, 8, 2653.
[15] Qiu, L.; Kwong, F. Y.; Wu, J.; Lam, W. H.; Chan, S. S.; Yu, W. Y.; Li, Y. M.; Guo, R. W.; Zhou, Z. Y.; Chan, A. S. C. J. Am. Chem. Soc. 2006, 128, 5955.

[16] Baeza, A.; Pfaltz, A. Chem. Eur. J. 2010, 16, 2036.

[17] Wang, D. S.; Chen, Q. A.; Li, W.; Yu, C. B.; Zhou, Y. G.; Zhang, X. M. J. Am. Chem. Soc. 2010, 132, 8909.

[18] Yang, S.-X.; Chen, J.; Wu, X.-Y.; Deng, H.-M.; Shao, M.; Zhang, H.; Cao, W.-G. Chin. J. Org. Chem. 2010, 30, 1521 (in Chinese). (杨树新, 陈杰, 吴小余, 邓红梅, 邵敏, 张慧, 曹卫国, 有机化 学, 2010, 30, 1521.)

[19] Rueping, M.; Brinkmann, C.; Antonchick, A. P.; Atodiresei, I. Org. Lett. 2010, 12, 4604.

[20] Duan, Y.; Chen, M. W.; Ye, Z. S.; Wang, D. S.; Chen, Q. A.; Zhou, Y. G. Chem. Eur. J. 2011, 17, 7193.

[21] Yip, K. T.; Yang, M.; Law, K. L.; Zhu, N. Y.; Yang, D. J. Am. Chem. Soc. 2006, 128, 3130.

[22] Fuller, P. H.; Kim, J. W.; Chemler, S. R. J. Am. Chem. Soc. 2008, 130, 17638.

[23] Yamamoto, H.; Ho, E.; Sasaki, I.; Mitsutake, M.; Takagi, Y.; Imagawa, H.; Nishizawa, M. Eur. J. Org. Chem. 2011, 2011, 2417.

[24] Nakanishi, M.; Katayev, D.; Besnard, C.; Kündig, E. P. Angew. Chem., Int. Ed. 2011, 50, 7438.

[25] Labadie, S. S.; Parmer, C. Synth. Commun. 2011, 41, 1752.

[26] Feltenberger, J. B.; Hsung, R. P. Org. Lett. 2011, 13, 3114.

[27] Viswanathan, R.; Prabhakaran, E. N.; Plotkin, M. A.; Johnston, J. N. J. Am. Chem. Soc. 2002, 125, 163.

[28] Viswanathan, R.; Smith, C. R.; Prabhakaran, E. N.; Johnston, J. N. J. Org. Chem. 2008, 73, 3040.

[29] Maciver, E. E.; Thompson, S.; Smith, M. D. Angew. Chem. 2009, 121, 10164.

[30] Kimura, M.; Futamata, M.; Mukai, R.; Tamaru, Y. J. Am. Chem. Soc. 2005, 127, 4592.

[31] Trost, B. M.; Quancard, J. J. Am. Chem. Soc. 2006, 128, 6314.

[32] Trost, B. M.; Zhang, Y. Chem. Eur. J. 2010, 16, 296.

[33] Franckevičius, V.; Cuthbertson, J. D.; Pickworth, M.; Pugh, D. S.; Taylor, R. J. K. Org. Lett. 2011, 13, 4264.

[34] Deboves, H. J. C.; Hunter, C.; Jackson, R. F. W. J. Chem. Soc., Perkin Trans. 1 2002, 733.

[35] Minatti, A.; Buchwald, S. L. Org. Lett. 2008, 10, 2721.

[36] Li, J. J.; Mei, T. S.; Yu, J. Q. Angew. Chem., Int. Ed. 2008, 47, 6452.

[37] Guthrie, D. B.; Curran, D. P. Org. Lett. 2009, 11, 249.

[38] Bruch, A.; Fröhlich, R.; Grimme, S.; Studer, A.; Curran, D. P. J. Am. Chem. Soc. 2011, 133, 16270.

[39] Sirasani, G.; Andrade, R. B. Org. Lett. 2011, 13, 4736.

[40] Hélène, P. Tetrahedron 2006, 62, 1619.

[41] Kawasaki, T.; Ogawa, A.; Takashima, Y.; Sakamoto, M. Tetrahedron Lett. 2003, 44, 1591.

[42] García Ruano, J. L.; Alemán, J.; Catalán, S.; Marcos, V.; Monteagudo, S.; Parra, A.; del Pozo, C.; Fustero, S. Angew. Chem., Int. Ed. 2008, 47, 7941.

[43] Yip, K. T.; Yang, D. Chem. Asian J. 2011, 42, 2166. 